\title{
Performance of a liquid argon time projection chamber exposed to the CERN West Area Neutrino Facility neutrino beam
}

F. Arneodo, ${ }^{1}$ P. Benetti, ${ }^{2}$ M. Bonesini, ${ }^{3}$ A. Borio di Tigliole,${ }^{2}$ B. Boschetti, ${ }^{3}$ A. Bueno, ${ }^{4}$ E. Calligarich, ${ }^{2}$ F. Casagrande,${ }^{5, *}$ D. Cavalli, ${ }^{3}$ F. Cavanna, ${ }^{1}$ P. Cennini, ${ }^{6}$ S. Centro, ${ }^{7}$ E. Cesana, ${ }^{2}$ D. Cline,${ }^{8}$ A. Curioni, ${ }^{3, \dagger}$ I. De Mitri, ${ }^{1}$ C. De Vecchi, ${ }^{2,7}$ R. Dolfini, ${ }^{2}$ A. Ferrari, ${ }^{6}$ A. Ghezzi, ${ }^{3}$ A. Guglielmi, ${ }^{7}$ J. Kisiel, ${ }^{9}$ G. Mannocchi ${ }^{10}$ A. Martínez de la Ossa, ${ }^{4}$ C. Matthey, ${ }^{8}$ F. Mauri, ${ }^{2}$ C. Montanari, ${ }^{2}$ S. Navas, ${ }^{4}$ P. Negri,${ }^{3}$ M. Nicoletto, ${ }^{7}$ S. Otwinowski ${ }^{8}$ M. Paganoni, ${ }^{3}$ O. Palamara,${ }^{11}$ A. Pepato, ${ }^{7}$ L. Periale, ${ }^{10}$ G. Piano Mortari, ${ }^{1}$ P. Picchi,${ }^{5,12}$ F. Pietropaolo, ${ }^{7}$ A. Puccini, ${ }^{6}$ A. Pullia, ${ }^{3}$ S. Ragazzi,${ }^{3}$ T. Rancati, ${ }^{3}$ A. Rappoldi, ${ }^{2}$ G. L. Raselli, ${ }^{2}$ N. Redaelli, ${ }^{3}$ E. Rondio, ${ }^{13}$ A. Rubbia, ${ }^{14}$ C. Rubbia, ${ }^{2}$ P. R. Sala, ${ }^{3}$ F. Sergiampietri, ${ }^{15}$ J. Sobczyk, ${ }^{16}$ S. Suzuki, ${ }^{5,12, *}$ T. Tabarelli de Fatis ${ }^{3}$ M. Terrani, ${ }^{2}$ F. Terranova, ${ }^{3}$ A. Tonazzo, ${ }^{3}$ S. Ventura, ${ }^{7}$ C. Vignoli, ${ }^{2}$ H. Wang, ${ }^{8}$ and A. Zalewska ${ }^{17}$

(The ICARUS-Milano Collaboration)

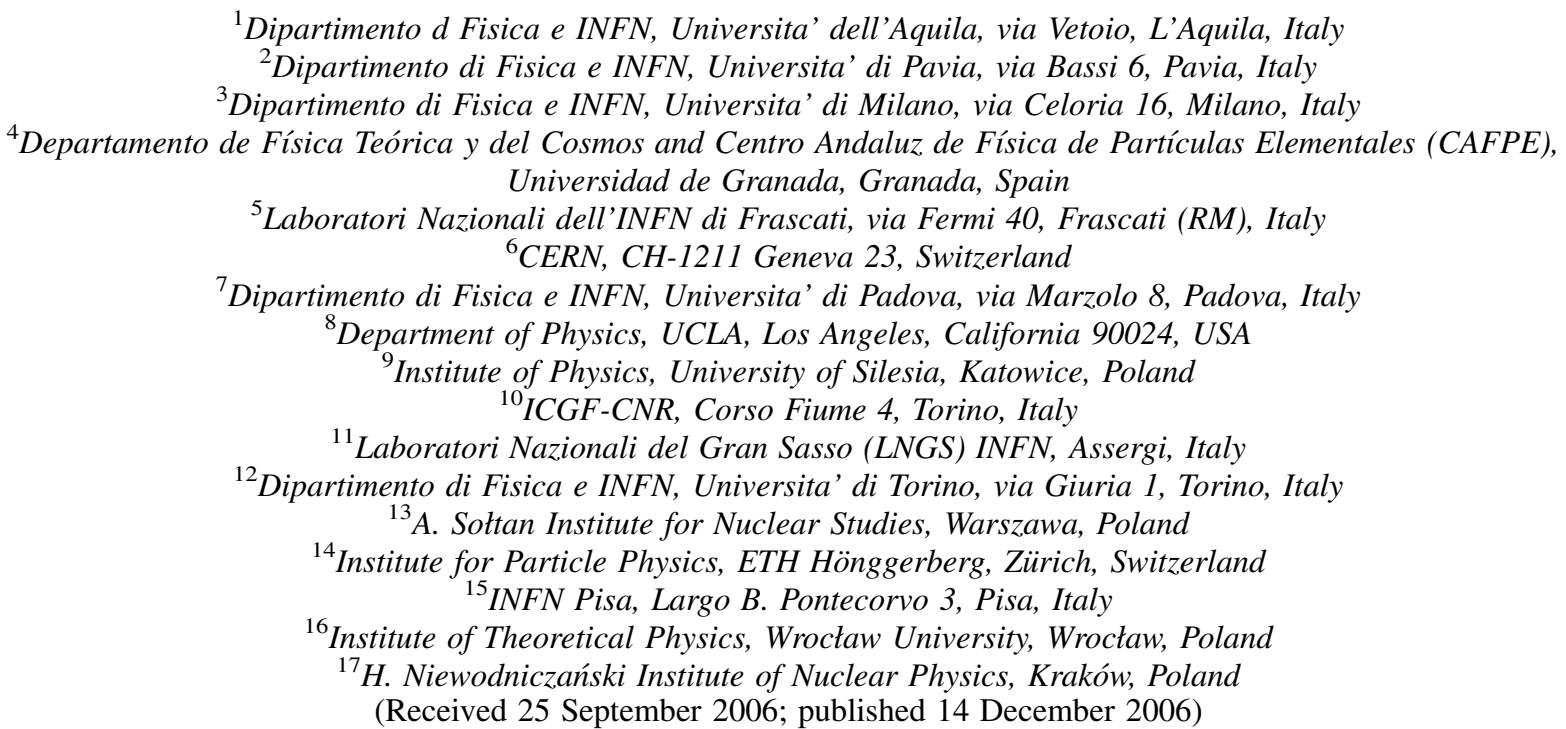

We present the results of the first exposure of a Liquid Argon TPC to a multi-GeV neutrino beam. The data have been collected with a 50 liters ICARUS-like chamber located between the CHORUS and NOMAD experiments at the CERN West Area Neutrino Facility (WANF). We discuss both the instrumental performance of the detector and its capability to identify and reconstruct low-multiplicity neutrino interactions.

DOI: 10.1103/PhysRevD.74.112001

PACS numbers: 29.40.Gx, 13.15.+g

\section{INTRODUCTION}

The Liquid Argon Time Projection Chamber (LAr TPC) technology was originally proposed by C. Rubbia in 1977 [1], as a novel concept for a massive neutrino detector that allows three-dimensional event reconstruction with high accuracy and precise calorimetric measurement. The feasibility of the technology, developed inside the context of the ICARUS project [2], has been demonstrated by an extensive research and development program that addressed the main technological challenges of the LAr

\footnotetext{
*Present address: Oakridge National Laboratory.

${ }^{\dagger}$ Present address: Yale University.

${ }^{\ddagger}$ Present address: Waseda University.
}

detection technique (i.e. argon purification, wire chambers, electronics, data acquisition (DAQ), etc.). The ICARUS collaboration has been able to operate prototypes of increasing mass ( 3 tons [3,4], 14 tons [5], 600 tons [6]), and therefore the LAr TPC can now be considered as a mature technology able to produce multikton detectors for astroparticle and neutrino physics.

Within the broad physics programme of the ICARUS project, an important issue is the study of oscillations through the detection of long-baseline neutrino interactions. The possibility to observe $\nu_{\mu} \rightarrow \nu_{e}$ and $\nu_{\mu} \rightarrow \nu_{\tau}$ by means of kinematic criteria is known to be limited, among others, by the knowledge we have of nuclear effects (Fermi motion, nuclear rescattering and absorption, etc.). Therefore it is very important to acquire experimental data 
in order to tune the existing Monte-Carlo models. In addition, the exposure of a LAr TPC to a neutrino beam is mandatory to demonstrate the high recognition capability of the technique and gain experience with real neutrino events.

In 1997, the ICARUS collaboration together with a group from INFN and Milano University [7] proposed to expose a 501 LAr TPC to the multi-GeV wide band neutrino beam of the CERN West Area Neutrino Facility (WANF) [8], during the NOMAD [9], and CHORUS [10] data taking. The test was part of an $R \& D$ program for a medium baseline $\nu_{\tau}$ appearance experiment [11]. The idea was to collect a substantial sample of quasielastic interactions $\left(\nu_{\mu}+\mathrm{n} \rightarrow \mathrm{p}+\mu^{-}\right)$to study the following physics items:

(i) Measurement of the acoplanarity and missing transverse momentum in events with the $\mu$-p topology in the final state, in order to assess Fermi motion and proton rescattering inside the nucleus.

(ii) Appearance of nuclear fragments (short tracks and blobs around the primary interaction vertex) in quasielastic events.

(iii) A preliminary evaluation of $e / \gamma$ and $e / \pi^{0}$ discrimination capability by means of the specific ionization measured on the wires at the beginning of the candidate track. This measurement is limited by the size of the chamber.

The data collected in 1997 offer the unique opportunity to study nuclear effects in the Argon nucleus and to assess the identification and reconstruction capability of a LAr TPC for low-multiplicity neutrino events. The present work shows, for the first time, a comprehensive set of results from the 1997 test (see [12-15] for preliminary results). The structure of the paper is as follows: the experimental setup and the instrumental performance of the 50 liters TPC are discussed in Sec. II and III, respectively. In Sec. IV the event reconstruction and particle identification are detailed. The analysis of a "golden sample" of quasielastic (QE) $\nu_{\mu}$ CC interactions is presented in Sec. $\mathrm{V}$ together with a comparison with theoretical expectations.

\section{THE EXPERIMENTAL SETUP}

The LAr TPC was placed on a platform 4.5 meters above ground, right in between the CHORUS and NOMAD detectors (Fig. 1). The modest size of the LAr TPC fiducial volume ( $\sim 50$ liters), coupled with the high energy of the WANF $\nu$ beam, made necessary a muon spectrometer downstream the TPC. A coincidence with the NOMAD DAQ was set up to use the detectors located into the NOMAD magnetic dipole as a magnetic spectrometer. The experimental setup was completed with additional counters for the trigger and veto systems.

The LAr TPC (Fig. 2) has an active volume of $32 \times$ $32 \times 46.8 \mathrm{~cm}^{3}$, enclosed in a stainless steel vessel in the

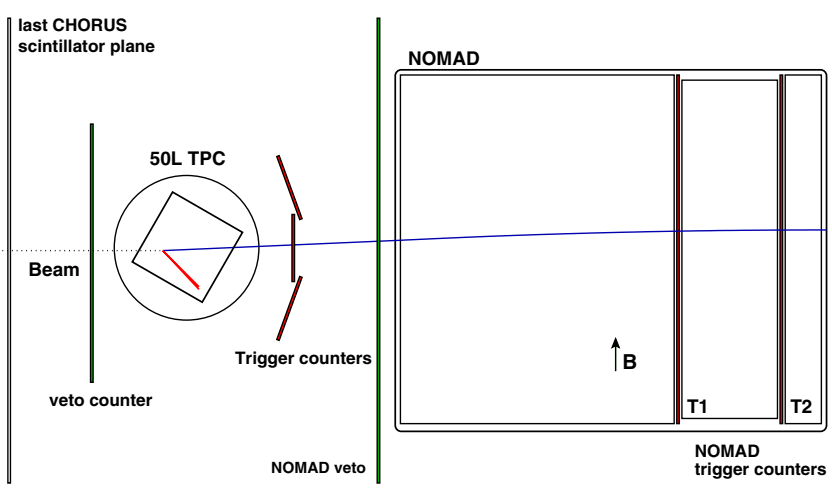

FIG. 1 (color online). A sketch of the experimental setup (top view). Relative sizes are not to scale.

shape of a bowed-bottom cylinder $90 \mathrm{~cm}$ high and with a radius of $35 \mathrm{~cm}$. The active volume corresponds to $67 \mathrm{~kg}$ of Liquid Argon $\left(T=87 \mathrm{~K}\right.$ at 1 atm, $\left.\rho=1.395 \mathrm{~g} / \mathrm{cm}^{3}\right)$. Ionization electrons produced by the passage of charged particles drift vertically toward the anode by means of a constant electric field of $214 \mathrm{~V} / \mathrm{cm}$. The read-ut area (anode) is made up of two orthogonal wire planes mounted at a distance of $4 \mathrm{~mm}$. Each stainless steel wire has a diameter of $100 \mu \mathrm{m}$; the distance between the wires is $2.54 \mathrm{~mm}$ and each plane is made up of 128 wires. The cathode and the lateral field-shaping electrodes are copper strips (5 mm thickness) positioned on a vetronite support with printed board techniques. The support was glued on a honeycomb structure to ensure rigidity. The distance between two adjacent strips is $10 \mathrm{~mm}$. The $10 \mathrm{KV}$ drift voltage is distributed to the strips by means of $100 \mathrm{M} \Omega$ resistors. The ionization electrons drift through the first wire plane (induction plane) so that the integrated signal induced in it is zero. The second plane (collection plane) stops the electrons and acts as charge collector. The mean charge per unit pitch $(2.54 \mathrm{~mm})$ for a mip crossing the chamber horizontally corresponds to about $2.3 \times 10^{4}$ electrons ( $\sim 4 \mathrm{fC}$ ) assuming no charge loss along the drift volume. The smallness of the signal and the absence of an amplification phase requires very low noise charge preamplifiers. They were based on JFET transistors and have been operated into the liquefied gas in the proximity of the readout wires. The polarization voltage $(15 \mathrm{~V})$ is distributed separately to each preamplifier to minimize the number of dead channels in case of failure of one of the components during data taking. The output signal is transmitted up to the amplifiers, which have been operated in current mode and are located at room temperature. The amplified signal is brought up to a set of fast 8-bit ADC. This configuration [12] allowed a signal to noise ratio of 11 with mip signals equivalent to $10 \mathrm{ADC}$ counts and no saturation even in the occurrence of e.m. showers. The signal is sampled with a $2.5 \mathrm{MHz}$ frequency for a duration of $500 \mu \mathrm{s}$, corresponding to the highest possible drift time (primary ionization at the cathode). The stream is recorded into a buffer. The arrival of a subsequent trigger causes the 

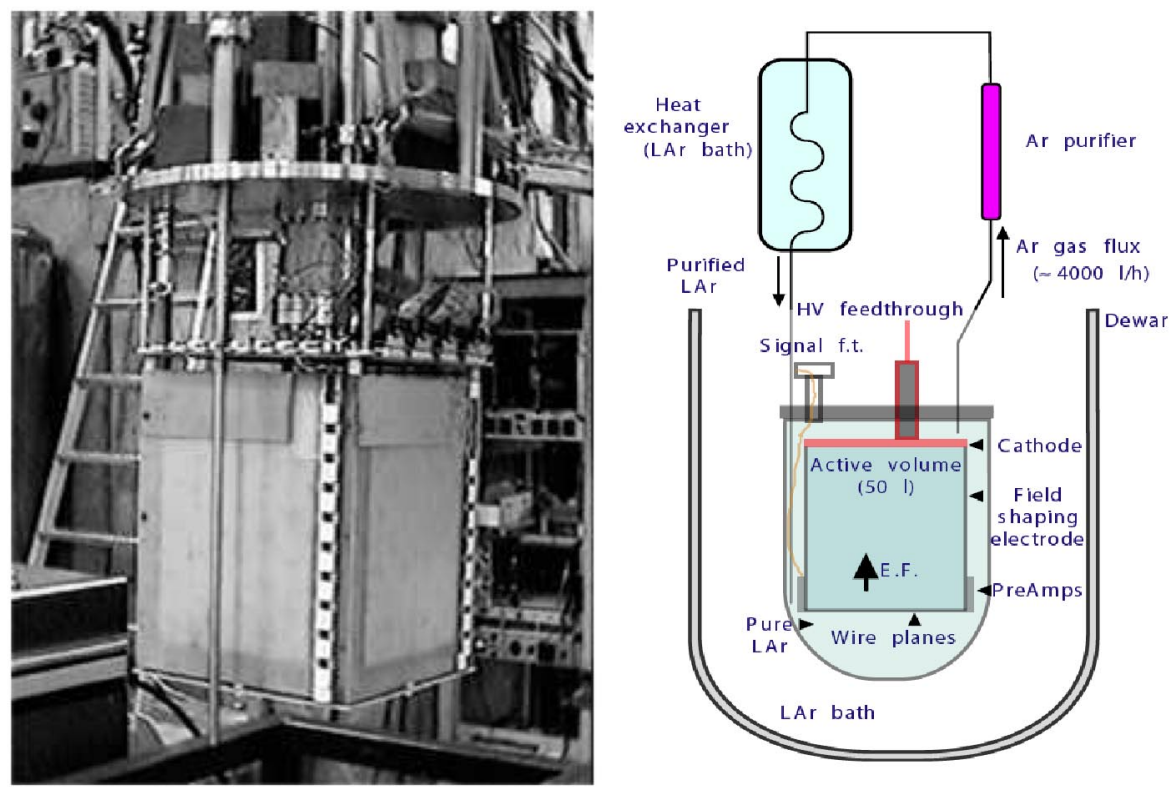

FIG. 2 (color online). The 50 liters Liquid Argon Time Projection Chamber.

switch of the data stream to another buffer (up to 8 buffers are available). The multibuffer writing procedure minimize the dead time for signal recording to less than one sampling time (i.e. $500 \mu$ s).

As mentioned above, the active part of the detector is located inside a stainless steel cylinder. The connection to the outside area is obtained through a set of UHV flanges housing the signal, the high voltage cables and the vacuum feed-through. The cylinder is positioned into a $1 \mathrm{~m}$ diameter dewar partially filled with low-purity Liquid Argon acting as a thermal bath and it is rotated $30^{\circ}$ along the vertical axis with respect to the nominal beam direction to reduce the number of particles crossing just one readout wire. The ceiling of the external dewar is in direct contact with air. The level of Argon in the dewar is so small that the pure $\mathrm{Ar}$ in the inner cylinder can evaporate as well. The gaseous $\mathrm{Ar}$ in the active volume crosses the feed-through and reaches an Oxisorb filter for purification [4]. After, it is liquefied inside a second buffer cooled by low-purity $\mathrm{Ar}$ and, finally, recollected into the active volume. During this test, the Argon has been doped with Tetramethyl Germanium in order to partially reconvert scintillation light into ionization [16]. Continuous recirculation of the Argon through the purifier keeps the level of contamination stable against micro-leaks or outgassing. The total Argon consumption necessary to circulate the pure liquefied gas and to compensate for the heat losses was $\sim 200$ liters per day. The performance of the recirculation system is described in Sec. III.

The chamber has been exposed to the $\nu$ beam produced at the CERN West Area Neutrino Facility (WANF $[8,17]$ ). The primary $450 \mathrm{GeV}$ protons from the CERN SPS are extracted every $14.4 \mathrm{~s}$ in two spills of $6 \mathrm{~ms}$ duration each and separated by $2.5 \mathrm{~s}$. On average, $1.8 \times 10^{13}$ protons per spill hit a segmented beryllium target. Secondaries are selected in momentum and focused by a system of collimators and magnetic lenses. They reach a $289.9 \mathrm{~m}$ decay tunnel followed by an iron and earth absorber. The experimental area is located $835 \mathrm{~m}$ downstream the target. The mean energy of the $\nu_{\mu}$ reaching the detectors is $24.3 \mathrm{GeV}$ when integrating over the active NOMAD area $(2.6 \times$ $2.6 \mathrm{~m}^{2}$ ), while contaminations from other flavors are below $7 \%$ for $\bar{\nu}_{\mu}$ and $\sim 1 \%$ for $\nu_{e}$ [17]. Being the TPC centered on the beam axis and covering a smaller surface, an harder neutrino flux is expected (with a mean energy of about $30 \mathrm{GeV}$ ).

The trigger is provided by a set of scintillators located between the chamber and the NOMAD apparatus (Fig. 1). Each of the 3 trigger scintillator counters has a dimension of $110 \times 27 \mathrm{~cm}^{2}$. They are positioned in a half-circle $60 \mathrm{~cm}$ beyond the center of the chamber. Incoming charged particle are vetoed by 5 scintillators mounted in front of the chamber, $50 \mathrm{~cm}$ before its center, and by the last scintillator plane of CHORUS. The latter vetoes particles deflected by the CHORUS magnetic field entering the chamber at large angles with respect to the nominal beam direction.

The trigger requires the coincidence of the SPS beam spill and at least one of the trigger scintillators, vetoed by the scintillators put in front of the chamber and the CHORUS plane. This trigger is put in coincidence with the two trigger scintillator planes of NOMAD (T1 and T2 in Fig. 1) [18]. Moreover, a trigger is rejected if the NOMAD acquisition system is in BUSY mode or if the delay with respect to the previous trigger is lower than $500 \mu \mathrm{s}$ ("drift protection"). ${ }^{1}$ The kinematic reconstruc-

\footnotetext{
${ }^{1}$ This condition inhibits the occurrence of event overlaps in the multibuffer readout system.
} 
tion of the outgoing muon from NOMAD (see Sec. IV D) allowed us to identify the events produced outside the fiducial volume that trigger the chamber. The vast majority are $\nu$ interactions in the dewar or in the thermal bath plus a contamination of crossing muons due to veto inefficiencies. Note that the request of a charged particle triggering the chamber locally and reaching NOMAD up to T1 and T2 inhibits the acquisitions of neutral-current and $\nu_{e}$ charged-current events, limiting the sample of the present test to $\nu_{\mu}$ charged-current interactions. The trigger efficiency has been monitored during data taking through a dedicated sample of through-going muons. It turned out to be $97 \%$ averaged over the whole data taking period.

\section{INSTRUMENTAL PERFORMANCE OF THE 50 LITERS TPC}

\section{A. Liquid argon purification and electron lifetime}

A crucial working parameter for a TPC operating with liquefied rare gases is the lifetime of free electrons in the medium. Primary electrons produced by the passage of charged particles drift toward the anode crossing macroscopic distances. In the present case, for an ionization electron created in the proximity of the cathode, the drift path-length exceeds $46 \mathrm{~cm}$. The effectiveness of charge collection at the readout plane is related to the purity of the Argon since electron-ion recombination is mainly due to oxygen molecules present in the LAr bulk [19]. The contamination of electronegative molecules must be at the level of $0.1 \mathrm{ppb}$ to allow drifts over $\mathcal{O}(1)$ meters. The electron lifetime $\tau_{e}$ is defined by:

$$
Q(t)=Q_{0} e^{-\left(t-t_{0} / \tau_{e}\right)}
$$

$Q_{0}$ being the primary deposited ionization charge and $t$ the electron drift time. The $t_{0}$ is provided by the trigger scintillation counters.

The effectiveness of the purification system through direct measurements of $\tau_{e}$ has been monitored during data taking by a dedicated setup first developed by the ICARUS Collaboration in 1989 [20]. It consists of a small double-gridded drift chamber located below the readout planes. Electrons are generated near the cathode via photoelectric effect driven by a $20 \mathrm{~ns}$ UV laser pulse. Each pulse produces a bunch of $10^{7}$ electrons. They drift toward the anode along the electric field lines and cross a $50 \mathrm{~cm}$ drift region between the two transparent grids. The ratio of the induced signal in the proximity of the grids provides a real time estimate of $\tau_{e}$ during data taking and, hence, a measurement of the Ar contamination (see Fig. 3). An independent estimate of $\tau_{e}$ can be obtained monitoring the charge attenuation for muons crossing the chamber at various heights. Both methods provided consistent results and the electron lifetime averaged along the whole data taking period turned out to be always higher than $10 \mathrm{~ms}$. This performance was excellent since the maximum drift

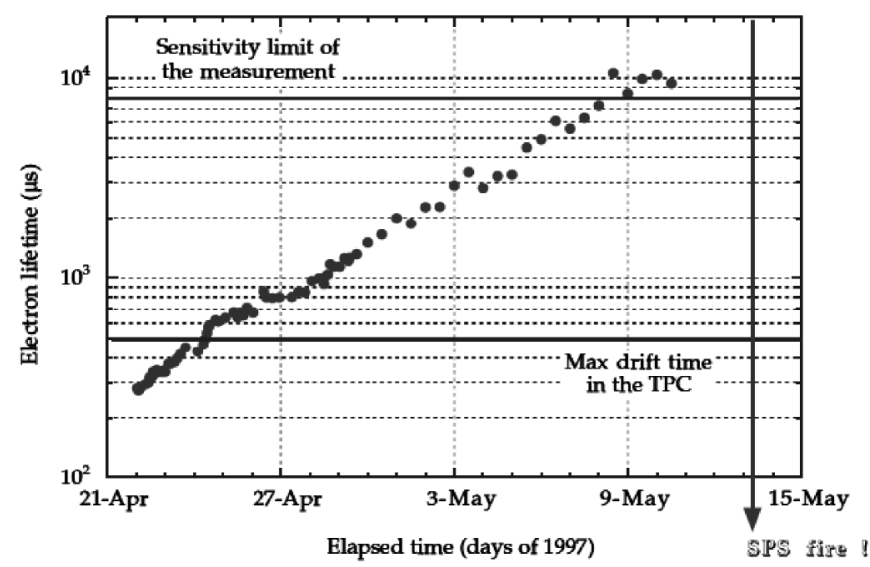

FIG. 3. Lifetime of the drifting electrons in the 50 liters Liquid Argon TPC at the CERN $\nu$ beam. The filling of the chamber with LAr was performed on the fourth of April 1997.

time was about $400 \mu \mathrm{s}$ and therefore the attenuation of the ionization over the drift distance was negligible.

\section{B. Drift velocity}

An absolute determination of the drift velocity and its uniformity along the fiducial volume of the chamber has been obtained exploiting the external scintillators and the additional information from NOMAD. A dedicated trigger selecting through-going muons ("mip sample") has been put into operation adding two additional scintillators $(30 \times$ $30 \mathrm{~cm}^{2}$ ) before and after the external dewar. The relative position of the chamber with respect to the NOMAD reference frame has been obtained by residual minimization of the track parameters recorded both by the TPC and the NOMAD data acquisition system [14]. The drift velocity is obtained by fitting the absolute vertical position of the muon as reconstructed by NOMAD trackers versus the drift time measured locally by the TPC. The corresponding drift velocity is $v_{d}=0.905 \pm 0.005 \mathrm{~mm} / \mu$ s (this value differs from the one quoted in [21] due to different running conditions of pressure and temperature). No systematic biases have been observed in the fiducial volume used for the neutrino interaction analysis (Sec. V).

\section{EVENT RECONSTRUCTION AND PARTICLE IDENTIFICATION}

\section{A. Spatial reconstruction}

Interactions happening in the TPC fiducial volume were fully registered in two 2D images with a common coordinate (time), with full calorimetric information associated to each point. Each 2D-image represents the signal amplitude digitized by the ADC (in a linear gray scale) versus the time sample (drift coordinate) and the wire number. Figure 4 and 5 (top part) show the raw images of two $\nu_{\mu}$ $\mathrm{CC}$ events in the collection (left panel) and induction plane (right panel). These raw images represent the time evolu- 


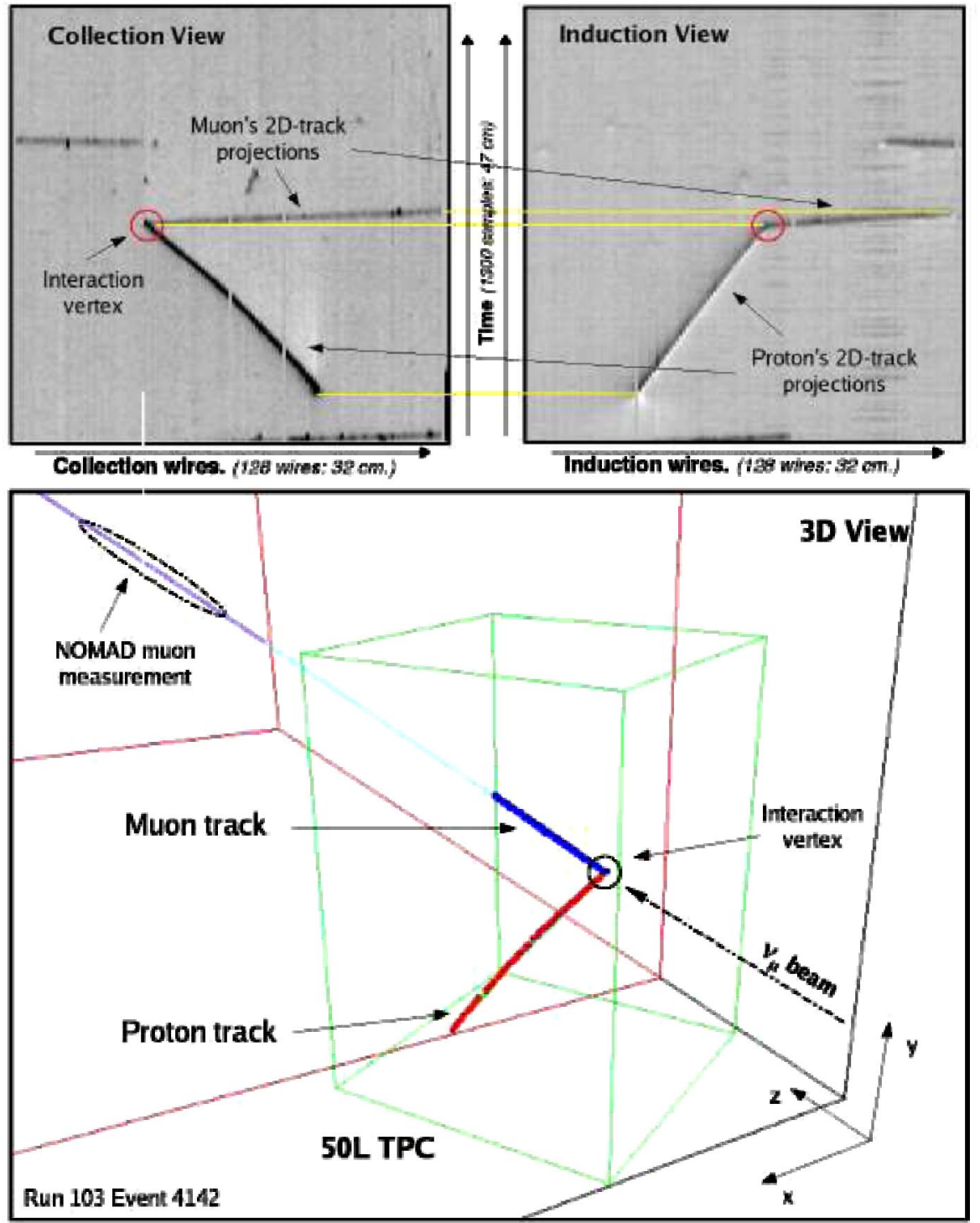

FIG. 4 (color online). Example of the 3D reconstruction of a low-multiplicity $\nu_{\mu} \mathrm{CC}$ event. The raw image from collection and induction wire planes (on top): Hits and 2D track projections have been identified. Three-dimensional view of the reconstructed event (bottom picture) embedded in a $3 \mathrm{D}$ recreation of the experimental setup described in Sec. II.

tion (in vertical axis) of the signal induced in the wires (in horizontal axis).

The purpose of the reconstruction procedure is to extract the physical information provided by the wire output signals, i.e. the energy deposited by the different particles and the point where such a deposition has occurred, to build a complete 3D and calorimetric picture of the event. In this sense, the spatial reconstruction of different tracks is needed first in order to compute the calibration factors entering in the calorimetric reconstruction of the events, as explained in Sec. IV B. A detailed description of the spatial reconstruction tools was reported elsewhere $[6,22]$. For the present analyses new significant improvements have been carried out [23].

The basic building block of a track is called a hit, defined as the segment of track whose energy is detected by a given wire. A precise determination of the hit position and charge is carried out fitting the wire signal to an analytical function which describes the detector response [6]. Identified nearby hits are associated into 2-dimensional clusters. Track candidates are searched for by means of a tree algorithm as in [24] and/or using a neural network-based algorithm as in [25]. Both methods efficiently detect interaction vertexes and finally, split clusters into smooth 2D tracks. Finally, 3D tracks are built matching 2D track projections from both views.

In Fig. 4, we show an example of the full reconstruction procedure for a low-multiplicity $\nu_{\mu} \mathrm{CC}$ event. This algorithm allows the reconstruction of more complicated event topologies (see Fig. 5). This is the first time that interactions of multi-GeV accelerator neutrinos occurring in a LAr TPC are fully reconstructed in 3D. 


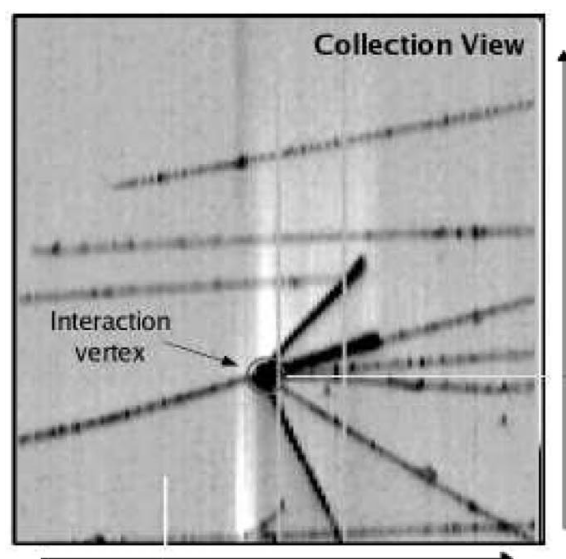

Collection wires. (128 wires: $32 \mathrm{~cm}$ )

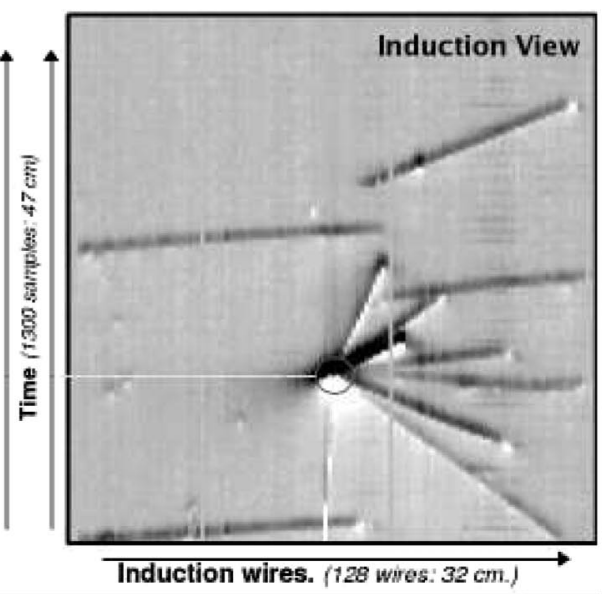

Induction wires. (128 wires: $32 \mathrm{~cm}$.)

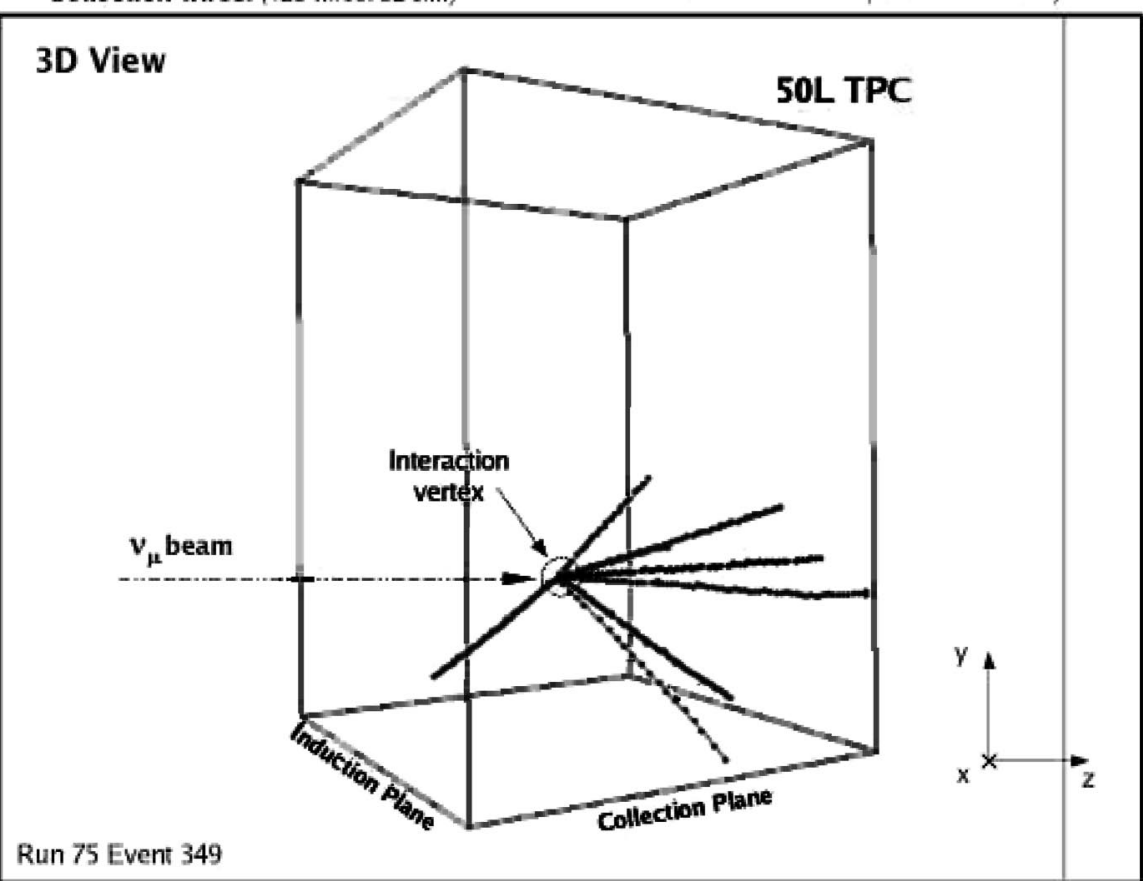

FIG. 5. (Top) The raw images of a high multiplicity event ( $\nu_{\mu}$ CC DIS) in the collection (left panel) and induction plane (right panel). Below, the 3D reconstruction of a high multiplicity event ( $\nu_{\mu}$ CC DIS $)$ with eight primary particles. Two of them stop in LAr and are recognized as protons.

\section{B. Calorimetric reconstruction}

The ionization charge is precisely measured at the collection wire plane. The energy $E$ associated to a given hit is

$$
E=\frac{C W}{R} Q(t)
$$

where $C$ is the calibration factor; $W$ is the average energy needed for the creation of an electron-ion pair (i.e. $23.6 \mathrm{eV}) ; R$ is the electron-ion recombination factor and $Q(t)$ is the corrected charge by the electron lifetime from (1) in Sec. III A.

The calibration factor $C$ converts the detector measuring units (ADC counts) into charge units (fC). To measure the overall calibration factor $\alpha \equiv \frac{C W}{R}$ in (2), we have collected a sample of around 3000 through-going muons coming from $\nu_{\mu} \mathrm{CC}$ interactions. The deposited charge per unit of length $(d Q / d x)$ is precisely measured (see Fig. 6) and fitted to a convoluted Landau-Gaussian function to obtain the most probable energy loss for mips in terms of detector charge units: $\langle d Q / d x\rangle_{\text {mip }}=216.7 \pm 0.1$ ADC counts. The most probable energy loss is a better defined quantity than the average value in the case of a Landau distribution, moreover it slightly depends on the energy in the range of the muons being considered. From this measurement, $\alpha$ can be obtained assuming that according to the theoretical prediction for muons in the range of energies of the beam, the most probable energy loss $\langle d E / d x\rangle_{\text {mip }}=1.736 \pm$ $0.002 \mathrm{MeV} / \mathrm{cm}$ : 


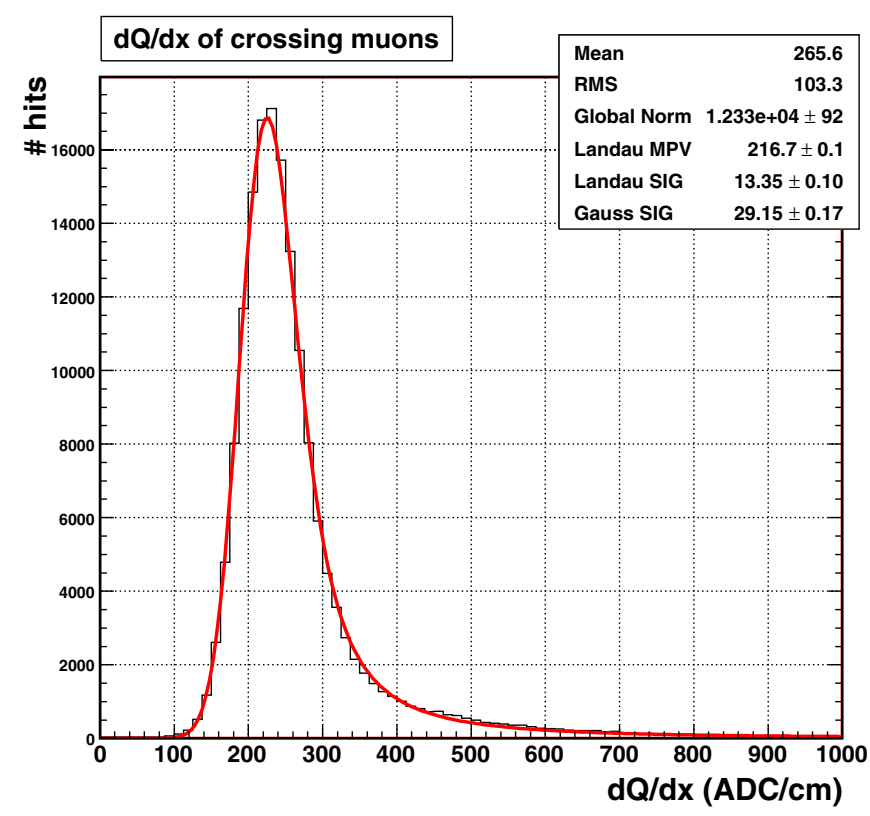

FIG. 6 (color online). $d Q / d x$ of crossing muons. The distribution is fitted to a convoluted Landau-Gaussian distribution.

$$
\begin{aligned}
\alpha_{\text {mip }} & \equiv \frac{C W}{R_{\text {mip }}}=\frac{\langle d E / d x\rangle_{\operatorname{mip}}}{\langle d Q / d x\rangle_{\operatorname{mip}}} \\
& =8.01 \pm 0.01 \times 10^{-3} \mathrm{MeV} / \mathrm{ADC}
\end{aligned}
$$

The recombination factor $R$ in (2) depends on the absorber medium, on the applied electric field and on the density of released charge, i.e. on $d E / d x$. This is the reason why we take in (3) $R_{\text {mip }}$ (its value for mips). The dependence of $R$ with $d E / d x$ can be modeled by Birk's law [26] which has been successfully applied to LAr detectors in [27]:

$$
\frac{d Q}{d x}=\frac{a d E / d x}{1+k_{B} d E / d x}
$$

$d E / d x$ being the pure Bethe-Bloch ionization loss, $a$ the energy to charge conversion factor and $k_{B}$ the Birks coefficient accounting for quenching due to recombination.

The size of the range of the fully contained protons collected in the "golden sample" considered in Sec. V, offers the opportunity to precisely test the energy loss pattern in the active medium. Figure 7 shows the observed charge per unit length $(d Q / d x)$ versus the residual range for the protons. ${ }^{2}$ Dots are direct measurements from the reconstructed hits of the proton tracks. From this distribution we estimate the most probable value of $d Q / d x$ for each bin of the residual range. Finally, from this data we fit

\footnotetext{
${ }^{2}$ The residual range is the actual range minus the range already covered at the time of the deposition of a given $d Q / d x$.
}

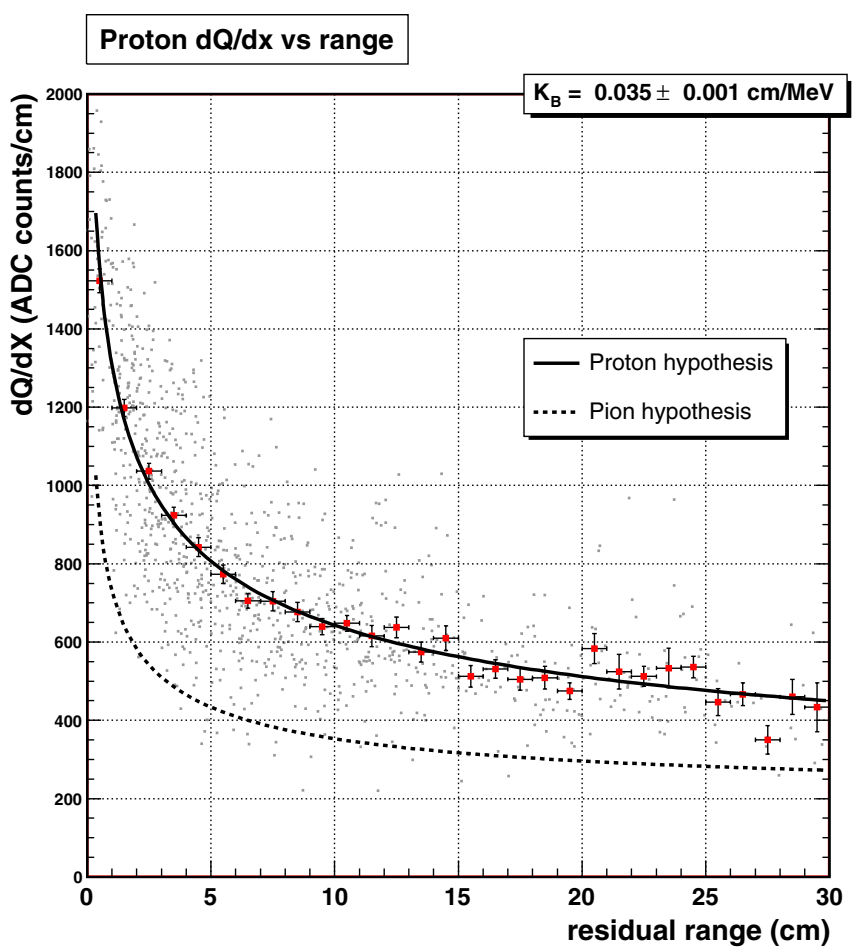

FIG. 7 (color online). $d Q / d x$ as a function of the residual range for protons. Dots are direct measurements from the reconstructed hits of the proton tracks, while the points with errors bars are the estimated most probable of $d Q / d x$ for each bin of the residual range. Data is fitted with a Bethe-Bloch corrected by the detector response function (Birk's law) in the hypothesis of pion (dashed line) and proton (full line).

$k_{B}=0.035 \pm 0.001 \mathrm{~cm} / \mathrm{MeV}$ in (4) for the proton hypothesis (continuous line).

Based on the knowledge of the calibration parameters, a precise determination of the deposited energy can be obtained through direct charge measurements by means of (4). Moreover, pure Bethe-Bloch ionization loss can be corrected by the detector response in the region of high $d Q / d x$, where quenching effects are more sizable. This correction is important for particle identification in LAr, which exploits the different energy loss of particles near the stopping point.

\section{Proton reconstruction}

Proton identification and momentum measurement were performed using only information provided by the TPC. The discrimination between protons and charged pions is performed exploiting their different energy loss behavior as a function of the range. For the case of candidates stopping in the fiducial volume of the chamber, as the ones of the "golden sample," $\mathrm{p} / \pi^{ \pm}$separation is unique (see Fig. 8 [left]): $d Q / d x$ is measured along the proton candidate track and is compared with the different particle hypothesis. Monte-Carlo studies reveal that almost $100 \%$ of protons are identified as such on the basis of their $d Q / d x$ 

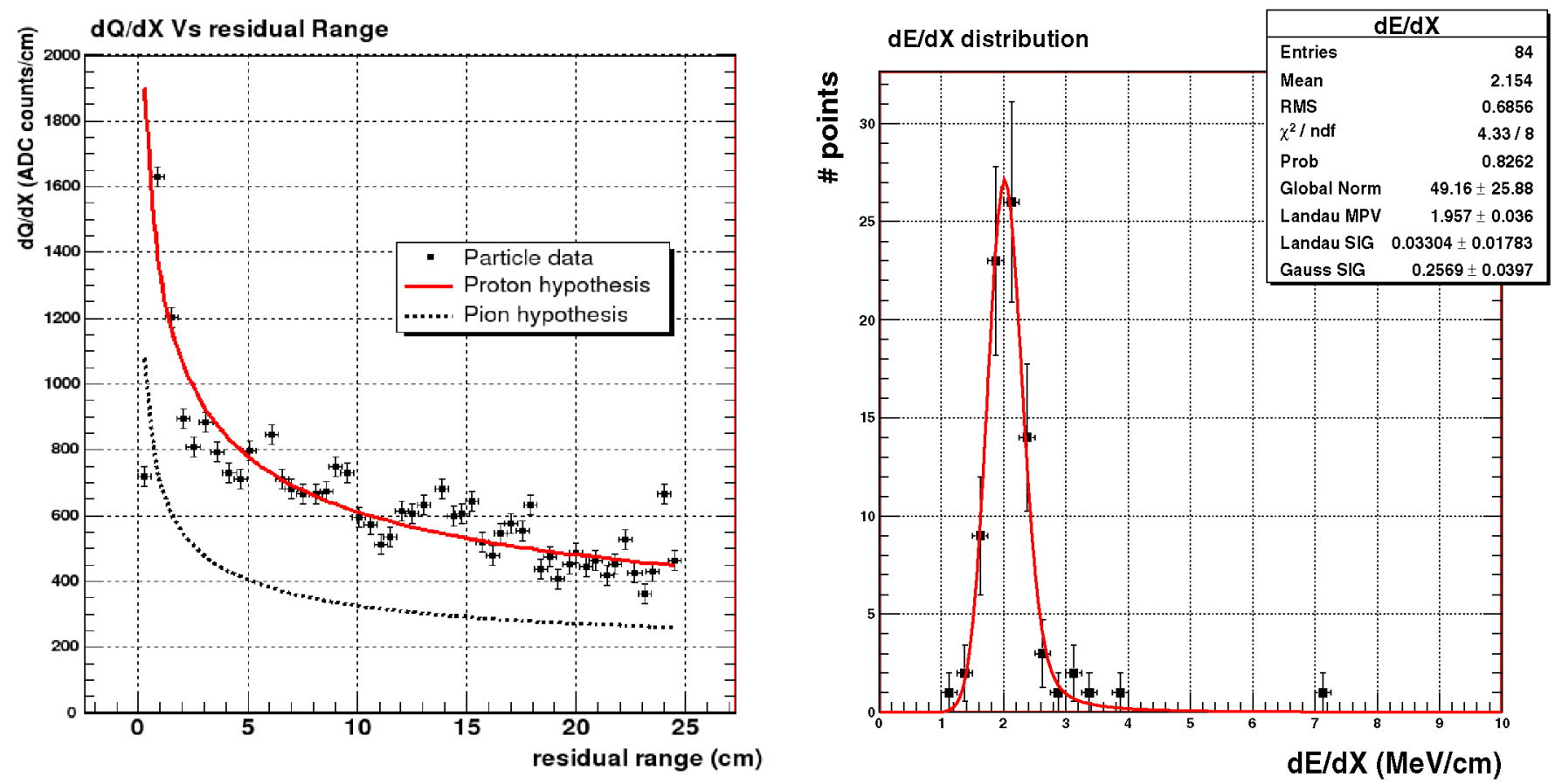

FIG. 8 (color online). (left) $d Q / d x$ as a function of the residual range for the reconstructed proton in the event shown in Fig. 4 : continuous (dashed) line represents the expected behavior of protons (pions) in the detector. (right) $d E / d x$ distribution for the muon reconstructed in Fig. 4: data is fitted to a convoluted Landau-Gaussian distribution.

shape on the vicinity of the stopping point; in addition, the fraction of pions and kaons misidentified as protons is negligible.

Once we identify a contained proton, its kinetic energy is calculated from range, which only depends on the spatial reconstruction of the tracks. In this case, the momentum uncertainty is dominated by the finite pitch of the wires $(2.54 \mathrm{~mm})^{3}$ and the performance of the reconstruction tools. These have been tested using a Monte-Carlo simulation of the detector. The resolution on the kinetic energy $\left(T_{p}\right)$ varies from $3.3 \%$ for protons with $T_{p}=50 \mathrm{MeV}$ up to $1 \%$ for $T_{p}$ larger than $200 \mathrm{MeV}$. The angular resolution i path; it is $\sigma \simeq 0.36 \sqrt{12} /\left(2.54 N^{3 / 2}\right)$, corresponding e.g. to $15 \mathrm{mrad}$ for $N=10$.

\section{Muon reconstruction}

The kinematic reconstruction of the outgoing muons exploits the tracking capability of NOMAD. An event triggering the chamber will have at least one penetrating track reaching the $\mathrm{T} 1$ and $\mathrm{T} 2$ trigger scintillators bracketing the TRD of NOMAD [18]. The corresponding track, nearly horizontal at the entrance of the NOMAD drift chamber volume, is reconstructed with an average momen-

\footnotetext{
${ }^{3}$ The equivalent pitch in the vertical direction (drift direction) is much smaller due to the high sampling rate of the fast ADC $(360 \mu \mathrm{m})$
}

tum precision of $\sigma_{p} / p \sim 0.05 / \sqrt{L} \oplus 0.008 p / L^{5 / 2}, \quad L$ being the visible range in the volume itself expressed in meters and $p$ the particle momentum in $\mathrm{GeV}$. A $10 \mathrm{GeV}$ horizontal muon crossing all the chambers $(L \sim 5 \mathrm{~m})$ is reconstructed with a precision of $2.2 \%$. The reconstructed particle is traced back to the TPC accounting for the magnetic field and the presence of the forward NOMAD calorimeter. The latter introduces an additional - in fact, dominant - source of uncertainty due to multiple scattering (MS) in iron (190 cm for a horizontal muon). For small scattering angles $(\theta \ll 1 \mathrm{rad})$, the MS uncertainty on the transverse momentum is independent of $p$ and it turns out to be $\sim 140 \mathrm{MeV}$. The correctness of the back-tracing procedure has been cross checked comparing the direction angles of the particles belonging to the mip sample, as measured by the TPC, with the corresponding quantity from NOMAD. ${ }^{4}$ Due to the large amount of material between the TPC and the NOMAD trackers (more than 11 interaction lengths) and the small distance between the chamber and the calorimeter, the $\pi / \mu$ misidentification probability is negligible for the present study, even accounting for possible $\pi$ decays in flight.

\footnotetext{
${ }^{4}$ Because of the high sampling rate of the TPC, the angular resolution in the $y-z$ plane, i.e. in the vertical plane along the nominal beam direction ( $z$ axis), is dominated by the NOMAD uncertainty.
} 


\section{ANALYSIS OF QUASI-ELASTIC $\nu_{\mu}$ INTERACTION}

\section{A. Data taking and event selection}

The data set recorded with the 50L chamber amounts to $1.2 \times 10^{19}$ protons on target. As mentioned in Sec. II, the trigger efficiency was monitored during data taking and its integrated value is $97 \%$. Additional losses of statistics are due to the TPC (3\%) and NOMAD (15\%) dead time and to detector faults. These contributions add up to give an effective lifetime of $75 \%$. Over the whole data taking period around 70000 triggers were collected in the TPC from which 20000 have at least a reconstructed muon possibly transversing the fiducial volume (see Sec. IV D). From all these a priory $\nu_{\mu} \mathrm{CC}$ candidates, a visual scanning reveals that around half of them show a vertex in the fiducial volume, the rest are $\nu$ interactions in the surrounding LAr bath (which also give trigger) plus a contamination of crossing muons due to veto inefficiencies. Therefore, we have a collection of around $10000 \nu_{\mu} \mathrm{CC}$ events from which we have selected a set of 86 events called the "golden sample." This set consists of events with an identified proton of kinetic energy larger than $40 \mathrm{MeV}$ fully contained in the TPC and one muon whose direction extrapolated from NOMAD matches the outgoing track in the TPC (see Sec. IV). The distance of the primary interaction vertex to any of the TPC walls has to be greater than $1 \mathrm{~cm}$. The muon candidate track projected onto the wire plane must be longer than 12 wire pitches. The event is accepted even in the presence of other stopping particles, as far as their visible range does not exceed the range of a proton of $40 \mathrm{MeV}$ kinetic energy. If other tracks than the identified muon leave the TPC or at least one converted photon with energy greater than $10 \mathrm{MeV}$ is present in the fiducial volume, the event is rejected. The tightness of these selections makes the "golden sample" a very clean topology for visual scanning (Fig. 4).

The request of containment is very severe since the chamber volume is small. For lower $T_{p}$ values, the proton range is comparable with the wire pitch and neither the proton momentum nor the interaction vertex can be reconstructed with due precision. However, for $T_{p}>40 \mathrm{MeV}$ the $\pi^{ \pm} / \mathrm{p}$ misidentification probability is negligible (see Sec. IV C). The "golden sample" contains pure QE interactions $\left(\nu_{\mu} n \rightarrow \mu^{-} p\right)$ plus an intrinsic background dominated by resonant production followed by pion absorption in the nucleus $\left(\nu_{\mu} p \rightarrow \Delta^{++} \mu^{-} \rightarrow \mu^{-} p \pi^{+}, \nu_{\mu} n \rightarrow\right.$ $\left.\Delta^{+} \mu^{-} \rightarrow \mu^{-} p \pi^{0}\right)$. There is also an instrumental background due to final state neutral particles $\left(\pi^{0}\right.$ s, $n$ 's, and $\gamma$ 's) that scape undetected. On the other hand, the $\nu_{\mu} n \rightarrow$ $\Delta^{+} \mu^{-} \rightarrow \mu^{-} n \pi^{+}$contamination is negligible in this tightly selected sample due to the superb $\pi^{ \pm} / \mathrm{p}$ identification capabilities of the LAr TPC.

\section{B. The Monte-Carlo data sample}

The selection efficiency for QE interactions and their intrinsic background have been evaluated using a MonteCarlo sample generated using the FLUKA package $[28,29]$, which offers a full description of nuclear effects in neutrino interactions. About $18000 \nu_{\mu} \mathrm{CC}$ QE events have been generated simulating the WANF beam for the $50 \mathrm{~L}$ chamber geometrical acceptance and final state particles were tracked in LAr using the GEANT4 package [30]. The energy deposition of all final particles is digitized emulating the TPC wire readout, in order to reproduce detector resolutions and offline reconstruction efficiencies (see Sec. IV). We saw that $16 \%$ of the whole sample of generated QE interactions belong to the "golden sample." On the other hand, we have analyzed a sample of $10^{5} \nu_{\mu}$ CC deep-inelastic (DIS) and resonant (RES) events in order to evaluate inefficiencies of the vetoing selections for $\nu_{\mu} n \rightarrow \Delta^{+} \mu^{-} \rightarrow \mu^{-} p \pi^{0}$ (i.e. the probability to miss both the decay photons of the $\pi^{0} \rightarrow \gamma \gamma$ or the $e^{+} e^{-} \gamma$ system in case of $\pi^{0}$ Dalitz decay) and for events with several neutral particles (neutrons and energetic photons from nuclear interactions) which scape from the detector (see Fig. 9). The search for this irreducible background in
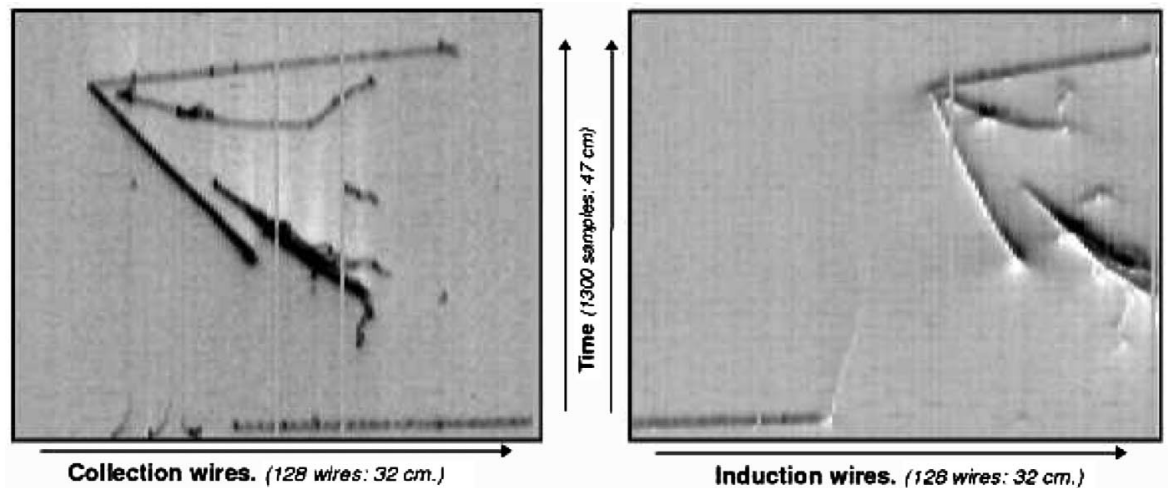

FIG. 9. The raw image of a low-multiplicity event in the collection (left) and induction plane (right). The event is reconstructed as $\left(\nu_{\mu} n \rightarrow \mu^{-} \Delta^{+} \rightarrow \mu^{-} p \pi^{0}\right)$ with a mip leaving the chamber, an identified stopping proton and a pair of converted photons from the $\pi^{0}$ decay. When these photons escape from the chamber, the event is tagged as a "golden event." 
the DIS plus RES samples reveals that $0.14 \%$ are "goldenlike" events.

Taking into account the relative weight between $\mathrm{QE}$ and non-QE events, ${ }^{5}$ we find that the total expected contamination of the "golden sample" is 20\% (around $40 \%$ of them correspond to events with an escaping $\pi^{0}$ ). The Monte-Carlo prediction for escaping $\pi^{0}$ can be checked using test samples that consist of golden events with one $\left(N_{1}\right)$ or two $\left(N_{2}\right)$ converted photons pointing to the interaction vertex. Assuming the gamma identification probability to be uncorrelated for the two photons, we have $N_{1}=2 N\left(1-\epsilon_{\gamma}\right) \epsilon_{\gamma}$ and $N_{2}=N \epsilon_{\gamma}^{2}, N$ being the (unknown) overall rate of $p \mu^{-} \pi^{0}$ final states and $\epsilon_{\gamma}$ the photon identification efficiency. After the scanning of the test samples, $\epsilon_{\gamma}=\left(N_{1} / 2 N_{2}+1\right)^{-1}$ turned out to be (43 \pm $9) \%$. Hence, the probability of missing both gammas is $(32 \pm 10) \%$ to be compared with the MC calculation of $20.4 \%$.

\section{Event rates}

We use Monte-Carlo to estimate the expected number of events in the detector. The simulation of the beam predicts a flux of $2.37 \times 10^{-7} \nu_{\mu} \mathrm{CC} / \mathrm{cm}^{2} /$ p.o.t. over the TPC exposing area. This flux convoluted with the neutrino cross sections and scaled to the fiducial mass of the detector gives an event rate of $2.05 \times 10^{-15} \nu_{\mu} \mathrm{CC} /$ p.o.t. Now assuming a total exposure of $1.2 \times 10^{19}$ p.o.t. and an effective lifetime of $75 \%$ (see Sec. VA), the total number of $\nu_{\mu}$ $\mathrm{CC}$ is equal to 18450 . Out of this 18450 events, only muons above a certain momentum threshold and within angular acceptance will trigger the detector. Taking the real data muons that triggered the system, we saw that most of them are above $8 \mathrm{GeV}$ in momentum and below $300 \mathrm{mrad}$ in angle. If we apply those cuts to the Monte-Carlo samples, we end up with $18450 \times 0.023(\mathrm{QE}$ fraction) $\times 0.95$ (efficiency of the acceptance cuts for QE), equal to 400 quasielastic events. For DIS + RES events: $18450 \times$ 0.977 (fraction of DIS + RES) $\times 0.65$ (efficiency of acceptance cuts) gives 11700 . In total we expect 12100 events to be compared with the $10000 \nu_{\mu} \mathrm{CC}$ we have after visual scanning of good triggers (see Sec. VA).

The expected number of "golden events" is obtained taking the 16\% (golden fraction) of the total QE, and adding the corresponding $20 \%$ due to background contribution (see Sec. VB), which finally gives $80 \pm 9$ (stat) \pm 13 (sys) "golden events" to be compared with the 86 we observe. The systematic uncertainty is dominated by the fraction of QE events $(2.3 \%)$ and the beam simulation $(8 \%)$.

\footnotetext{
${ }^{5}$ Respect to the total number of $\nu_{\mu} C C$ events, $2.3 \%$ are QE, $91.5 \%$ are DIS and the remaining $6.2 \%$ corresponds to resonances events. The simulation of the latest is not done treating each resonance individually, an average of them is taken into account instead.
}

\section{Analysis of quasielastic interactions}

In spite of the limited statistics (86 events), the "golden sample" provides information on the basic mechanisms that modify the kinematics of neutrino-nucleus interactions with respect to the corresponding neutrino-nucleon process. Nuclear matter perturbs the initial state of the interaction through Fermi motion; it also affects the formation of the asymptotic states through nuclear evaporation, hadronic rescattering or hadronic reabsorption. Several kinematic variables are only marginally affected by nuclear effects; in this case, the corresponding distributions can be reproduced once the $\nu$-nucleon interaction is corrected for Fermi motion and Pauli blocking. Clearly, purely leptonic variables belong to this category. There are also a few hadronic variables whose distribution is strongly influenced by the selection cuts but show limited sensitivity to nuclear effects. In particular, the proton kinetic energy is bounded by the $T_{p}>40 \mathrm{MeV}$ cut and the requirement of full containment in the fiducial volume. This distribution is shown in Fig. 10 together with the transverse momentum of the proton for the "golden sample." Similarly, in Fig. 11 we show the kinetic energy and the transverse momentum distributions of the muon. Figures 10 and 11 can be used as a consistency check. They demonstrate that Monte-Carlo reproduces the kinematic selection performed during the scanning and analysis of the "golden channel." They also show that our selected sample contains a non-negligible contamination from non-QE events. As already indicated, we estimate this contamination to be $20 \%$.

Other variables embedding the reconstructed kinematics of the protons are sensitive to genuine nuclear effects. In particular, we analyzed two of them: the acollinearity and the missing transverse momentum of the event. The former is defined as

$$
A \equiv \operatorname{acos}\left[\frac{p_{x p} p_{x \mu}+p_{y p} p_{y \mu}}{\sqrt{\left(p_{x p}^{2}+p_{y p}^{2}\right)\left(p_{x \mu}^{2}+p_{y \mu}^{2}\right)}}\right],
$$

$p_{x p}$ and $p_{y p}$ being the transverse momentum components of the proton and $p_{x \mu}$ and $p_{y \mu}$ the corresponding quantities for the muon. For a purely QE scattering on a nucleon, the muon and the proton ought to be back-to back in the transverse plane so that the acollinearity is zero. Figure 12 (right) shows the acollinearity distribution for the "golden sample" and the expectation from simulations. For this particular variable, the inclusion of nuclear effects in the Monte-Carlo does not show a striking difference with respect to the case where no nuclear effects are taken into account. The selection cuts (in particular, the fact that the proton should be fully contained), the resolution NOMAD has for muon reconstruction and the contamination from non-QE events are the main reasons for the appearance of events with large acollinearity values. The distortion introduced in the tail of the acollinearity distribution by nuclear effects is much smaller than the one 

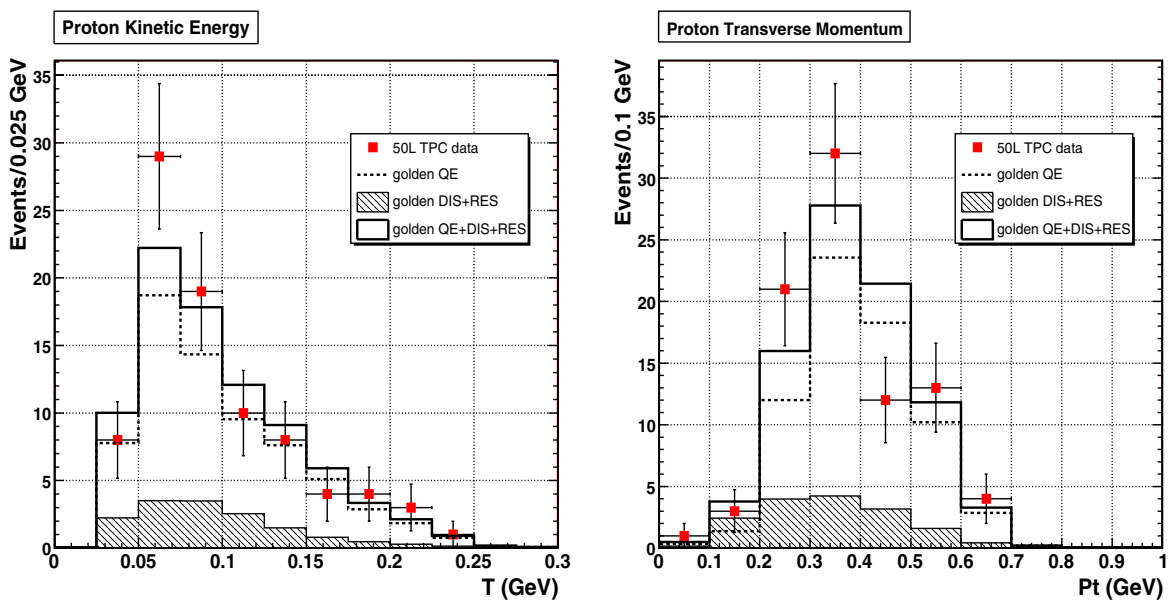

FIG. 10 (color online). Distribution of the proton kinetic energy for the "golden sample" (squared marks). Dotted histogram represents the expectation from simulated Monte-Carlo QE events, while the hatched one represents the simulated background from non-QE events. Both contributions are summed in the filled histogram and normalized to the golden sample data.
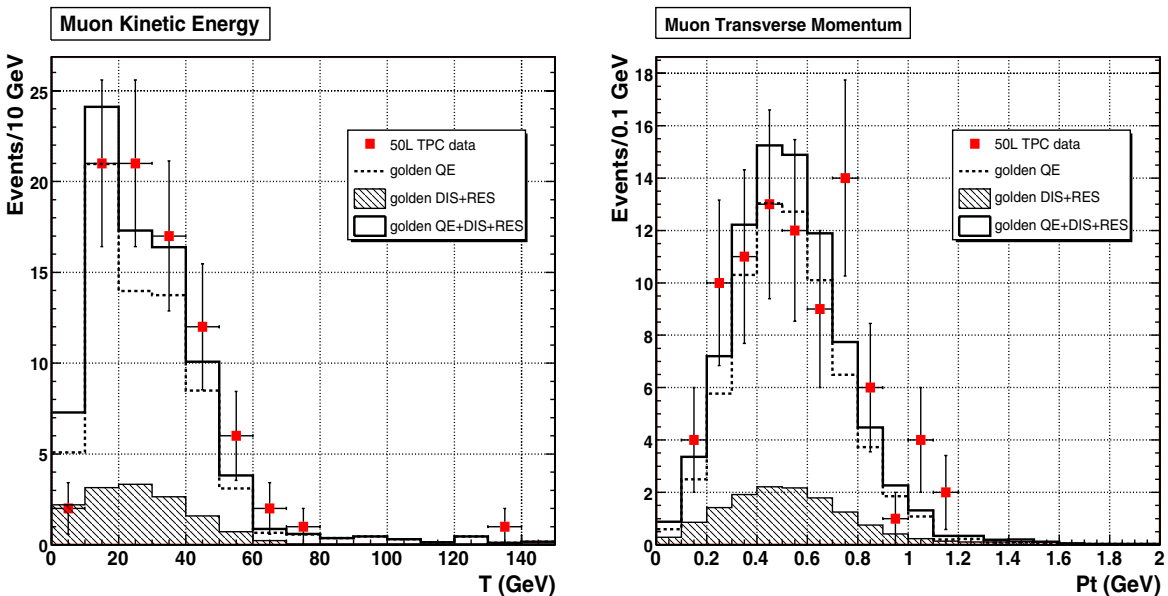

FIG. 11 (color online). Distribution of the muon energy and the muon transverse momentum for the "golden sample." Dotted histogram represents the expectation from simulated Monte-Carlo QE events, while the hatched one represents the simulated background from non-QE events. Both contributions are summed in the filled histogram and normalized to the golden sample data.
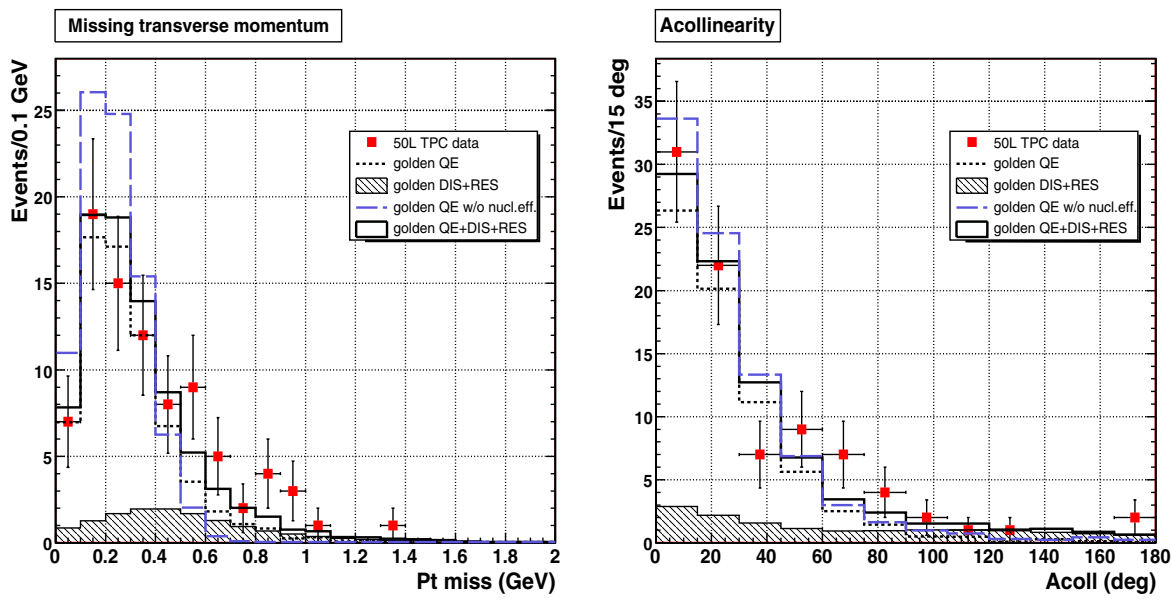

FIG. 12 (color online). Distribution of the missing transverse momentum (left) and acollinearity (right) for the "golden sample." Dotted histogram represents the expectation from simulated Monte-Carlo QE events, while the hatched one represents the simulated background from non-QE events. Both contributions are summed in the filled histogram and normalized to the golden sample data. The dashed histograms show the expected distributions in case no nuclear effects are taken into account. 
due to detector effects. Hence this variable is not adequate to show how the event kinematics varies in the presence of nuclear matter.

The influence of nuclear effects on the event kinematics is best seen when we consider the missing transverse momentum $p_{T}^{\text {miss }}$ (see left plot on Fig. 12). We observe that a naive approach that takes into account Fermi motion and Pauli blocking disregarding nuclear effects (dashed histograms in Fig. 12 left) does not reproduce the $p_{T}^{\text {miss }}$ data. Once nuclear effects are added, simulated events show a good agreement with data.

In summary, we have seen that nuclear effects are an important source of perturbation for the kinematics of quasielastic neutrino interactions, however the smallness of the accumulated statistics does not allow a systematic survey of these effects [31]. Still, these results empirically demonstrate the effectiveness of LAr imaging in the characterization of low-multiplicity multi-GeV neutrino interactions. In particular, a good description of transverse variables is fundamental since they are key to a kinematic-based approach for $\nu_{\mu} \rightarrow \nu_{\tau}\left(\nu_{e}\right)$ oscillation appearance [32].

\section{CONCLUSIONS}

We discussed the first exposure of a LAr TPC to a multi$\mathrm{GeV}$ neutrino beam. The data collected at the CERN WANF exploited the 3D reconstruction capabilities of the LAr technology with low-multiplicity events. The instrumental performances (size and stability of the electron lifetime, reproducibility of the charge response for mip and highly ionizing particles) and current offline reconstruction tools show that LAr TPC allows for an excellent particle identification and a precise measurement of the event kinematics. In spite of the limited size of the detector, nuclear effects beyond Fermi motion and Pauli blocking (e.g. hadron rescattering or pion absorption) have been observed as perturbations of the quasielastic $\nu_{\mu} \mathrm{CC}$ interaction kinematics. Variables in the transverse plane, which are the most sensitive to nuclear effects and key for neutrino oscillation searches, are well reproduced.

\section{ACKNOWLEDGMENTS}

We wish to express our gratitude to the members of the NOMAD and CHORUS collaboration for their support during the setting-up of the test and data taking. We specially thank the help of the NOMAD Collaboration which agreed to increase slightly its dead-time to include the new dedicated trigger needed to acquire the escaping muons. We acknowledge the CERN SPS accelerator and beamline staff for the excellent performance of the WANF neutrino beam. We thank the CERN PH Division since a large fraction of the LAr consumption costs were financially supported by it. The spanish group has been supported by M. E. C. through Grant No. FPA2002-01835. The Polish authors acknowledge the support of the State Committee for Scientific Research in Poland, 105, 160, 620, 621/E-344, E-340, E-77, E-78/SPS/ICARUS/P-03/ DZ211-214/2003-2005.
[1] C. Rubbia, CERN Report No. CERN-EP/77-08, 1977 (unpublished).

[2] ICARUS Collaboration, INFN Report No. INFN/AE-85/7, 1985 (unpublished); ICARUS-II. A second generation proton decay experiment and neutrino observatory at the Gran Sasso laboratory, Proposal No. LNGS-94/99, VOL I (1993) \& II (1994); A first T600 ton ICARUS detector installed at the Gran Sasso laboratory, Addendum to Proposal, LNGS 95-10 (1995).

[3] P. Bennetti et al., Nucl. Instrum. Methods Phys. Res., Sect. A 332, 395 (1993).

[4] P. Cennini et al., Nucl. Instrum. Methods Phys. Res., Sect. A 345, 230 (1994).

[5] F. Arneodo et al., Nucl. Instrum. Methods Phys. Res., Sect. A 498, 292 (2003).

[6] S. Amerio et al., Nucl. Instrum. Methods Phys. Res., Sect. A 527, 329 (2004).

[7] S. Ragazzi et al., SPSLC Report No. SPSLC/M 594, 1997 (unpublished).

[8] G. Acquistapace et al., CERN Report No. CERN-ECP/9514, 1995 (unpublished).
[9] J. Altegoer et al., Nucl. Instrum. Methods Phys. Res., Sect. A 404, 96 (1998).

[10] E. Eskut et al., Nucl. Instrum. Methods Phys. Res., Sect. A 401, 7 (1997).

[11] A. Rubbia et al., CERN-SPSLC Report No. CERNSPSLC-96-58/SPSLC-P-304 (unpublished).

[12] F. Arneodo et al., hep-ex/9812006.

[13] A. Curioni, Diploma thesis, Milano, 1997.

[14] B. Boschetti, Diploma thesis, Milano, 1997.

[15] A. Curioni (ICARUS-Milano Collaboration), Nucl. Phys. B, Proc. Suppl. 159, 69 (2006).

[16] P. Cennini et al., Nucl. Instrum. Methods Phys. Res., Sect. A 355, 660 (1995).

[17] P. Astier et al., Nucl. Instrum. Methods Phys. Res., Sect. A 515, 800 (2003).

[18] J. Altegoer et al., Nucl. Instrum. Methods Phys. Res., Sect. A 428, 299 (1999).

[19] A. Bettini et al., Nucl. Instrum. Methods Phys. Res., Sect. A 305, 177 (1991).

[20] G. Carugno et al., Nucl. Instrum. Methods Phys. Res., Sect. A 292, 580 (1990). 
[21] S. Amoruso et al. (ICARUS Collaboration), Nucl. Instrum. Methods Phys. Res., Sect. A 516, 68 (2004).

[22] J. Rico, Ph.D. thesis, Dissertation [Institution Report No. ETH 14906, 2002 (unpublished)]; Available at: http://neutrino.ethz.ch/diplomathesis.html.

[23] A. Martinez de la Ossa, Ph.D. thesis, Universidad de Granada, 2006.

[24] D. G. Cassel and H. Kowalski, Nucl. Instrum. Methods 185, 235 (1981).

[25] G. Stimpfl-Abele and L. Garrido, Comput. Phys. Commun. 64, 46 (1991).

[26] J. Birks, Theory and Practice of Scintillation Counting (Pergamon Press, New York, 1964).

[27] S. Amoruso et al. (ICARUS Coll.), Nucl. Instrum. Methods Phys. Res., Sect. A 523, 275 (2004).
[28] A. Fasso, A. Ferrari, J. Ranft, and P. R. Sala, CERN Report No. CERN-2005-10, INFN Report No. INFN/TC-05/11, SLAC Report No. SLAC-R-773, 2005 (unpublished).

[29] A. Fasso et al., hep-ph/0306267.

[30] S. Agostinelli et al., Nucl. Instrum. Methods Phys. Res., Sect. A 506, 250 (2003).

[31] For a review of the experimental perspectives to explore nuclear effects in this kinematic regime we refer to the Proceedings of the Third International Workshop on Neutrino-Nucleus Interactions in the Few-GeV Region, edited by F. Cavanna, T. Keppel, P. Lipari, M. Sakuda [Nucl. Phys. B (Proc. Suppl.) 439, No. 1 (2004).

[32] A. Bueno et al., J. High Energy Phys. 11 (2004) 14. 